\title{
Risk factors for contact lens-related microbial keratitis in Singapore
}

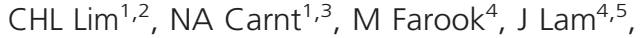
DT $\operatorname{Tan}^{4,5,6,7}$, JS Mehta ${ }^{4,7}$ and F Stapleton ${ }^{1}$

\begin{abstract}
Purpose: To investigate independent risk factors for contact lens-related microbial keratitis in Singapore and estimate their impact on disease load.

Methods: Cases were contact lens wearers presenting to Singapore National Eye Centre with microbial keratitis between 2008 and 2010. Community contact lens wearers were recruited as controls. All wearers completed a previously validated questionnaire describing contact lens wear history, hygiene and compliance habits, and demographics. Risk factors significant in univariate analysis $(P<0.2)$ were evaluated in a multivariate model.
\end{abstract}

Results: In all, 58 cases of microbial keratitis and 152 contemporaneous controls were identified. When controlling for other variables, Chinese had a $7 \times$ lower risk compared with other races (95\% CI: 2.3-21.3, $P=0.001$ ). Those aged between 25 and 44 years were at $3 \times$ increased risk compared with younger wearers (95\% CI: 1.1-9.6, $P=0.04)$. Occasional overnight contact lens wear (less often than one night per week) was associated with a $4 \times$ higher risk $(95 \%$ CI: 1.2-15.4, $P=0.03$ ) compared with daily use. Not washing hands before handling was associated with a $13 \times$ increased risk $(95 \% \mathrm{CI}$ : 1.9-84.8, $P=0.008$ ). Use of multipurpose solution A carried a $16 \times$ higher risk compared with hydrogen peroxide (95\% CI: 1.5-174.0, $P=0.02)$. The combined PAR $\%$ for modifiable risk factors (occasional overnight wear, not washing of hands, and MPS A) was $82 \%$. Conclusions: Consistent with previous findings, independent risk factors for contact lens-related microbial keratitis include poor hand hygiene, occasional overnight wear, and type of lens care solution. Prolonged overnight or extended contact lens use was infrequent in this population.

Eye (2016) 30, 447-455; doi:10.1038/eye.2015.250; published online 4 December 2015

\section{Introduction}

Contact lenses are a common means of reversible vision correction that offer cosmetic and optical advantages over spectacles. There are an estimated 140 million individuals who wear contact lenses worldwide. ${ }^{1}$

Complications of contact lens wear are generally self-limiting on lens removal with the exception of contact lens-related microbial keratitis, a rare but potentially sight-threatening condition. Epidemiological studies of contemporary contact lenses have estimated the incidence of microbial keratitis in daily wear at 3 to 5 per 10000 lens wearers per year, with incidence varying according to wear modality. ${ }^{2-6}$

Risk factors that have been consistently identified may be categorised into either modifiable or nonmodifiable risk factors. Modifiable risk factors include extended and overnight contact lens wear, ${ }^{7,8}$ poor hand hygiene, ${ }^{4,9}$ infrequent or omission of lens disinfection, $, 7,8$ case hygiene and case replacement, ${ }^{6,10}$ and smoking. ${ }^{4,6,11}$ Nonmodifiable risk factors include gender, ${ }^{2,7,11}$ young age, ${ }^{2,7}$ and socioeconomic status. ${ }^{9}$ Systemic risk factors for contact lens-related microbial keratitis include poor general health, ${ }^{12}$ diabetes, ${ }^{9}$ and thyroid disease. ${ }^{12}$ More recently, lens supply has been implicated as a risk factor where internet or mail order supply of contact lenses has been associated with a higher risk of microbial keratitis compared with obtaining lenses through a contact lens practitioner. ${ }^{6,13}$

Factors identified as contributing to the severity of microbial keratitis include pathogenic or environmental microorganisms ${ }^{14,15}$ and high daytime temperatures, such as in tropical regions like Singapore. ${ }^{15}$

Patterns of contact lens prescribing, lens supply, wearer behaviour, and environmental microbiota vary across different cultures and climates. These may affect the risk of contact lens-related microbial keratitis. It is therefore
${ }^{1}$ School of Optometry and Vision Science, University of New South Wales, Sydney, NSW, Australia

${ }^{2}$ Royal Melbourne Hospital, Parkville, VIC, Australia

${ }^{3}$ Moorfields Eye Hospital Trust, London, UK

${ }^{4}$ Singapore National Eye Centre, Singapore, Singapore

${ }^{5}$ Department of Ophthalmology, National University Hospital, Singapore, Singapore

${ }^{6}$ Department of Ophthalmology, Yong Loo Lin School of Medicine, National University of Singapore, Singapore, Singapore

${ }^{7}$ Ophthalmology and Visual Sciences Academic Clinical Program, DukeNUS Graduate Medical School, Singapore, Singapore

Correspondence: F Stapleton, School of Optometry and Vision Science, University of New South Wales, Sydney, NSW 2052, Australia Tel: +61 2 93854375; Fax: +61 293136243. E-mail: f.stapleton@ unsw.edu.au

Received: 28 June 2015 Accepted in revised form: 21 October 2015 Published online: 4 December 2015 
important to examine specific populations to determine specific risk factors associated with the use of contact lenses in these populations. This study reports risk factors for contact lens-related microbial keratitis in Singapore and estimates the impact of these risk factors on disease load.

\section{Materials and methods}

\section{Case ascertainment}

New cases of contact lenses-related microbial keratitis were identified in a 32-month prospective study of wearers presenting to the Singapore National Eye Centre (SNEC), a tertiary ophthalmic referral centre, between January 2008 and August 2010.

The definition of microbial keratitis was chosen to align with previous reports of contact lens-related microbial keratitis. ${ }^{6,13,16}$ In brief, microbial keratitis was defined by either a positive corneal culture or a corneal infiltrate and overlying epithelial defect with ONE OR MORE of the following characteristics:

- Any part of the lesion within or overlapping the central $4 \mathrm{~mm}$ of the cornea

- Uveitis

- Pain

Written consent was obtained from cases to allow access to medical records and subjects completed a selfadministered questionnaire ${ }^{6,13,16,17}$ to establish potential risk factors, including contact lens type, wear habits, hygiene compliance and history, and demographics. Risk factors considered in the analysis are shown in Table 1. Human research ethics approval was obtained from the

Table 1 Risk factors

\begin{tabular}{|c|c|}
\hline Demographic information & Gender \\
\hline & Race \\
\hline & Age \\
\hline & Occupation \\
\hline & Education \\
\hline Lens wear history & Duration of contact lens wear \\
\hline & Lens replacement frequency \\
\hline & Wear schedule \\
\hline & Overnight wear \\
\hline Lens details & Type of lens used \\
\hline & Lens care solution used \\
\hline & Regular lens case replacement \\
\hline & Lens case hygiene \\
\hline Water exposure & Showering while wearing lenses \\
\hline & Time of day shower taken \\
\hline & Swimming while wearing lenses \\
\hline Hand hygiene & $\begin{array}{l}\text { Washing and/or drying hands before } \\
\text { handling lenses }\end{array}$ \\
\hline Other behavioural factors & Travel history over the past 3 months \\
\hline
\end{tabular}

University of New South Wales Human Research Ethics Committee and from Singapore National Eye Centre, and the study was conducted in accordance with the tenets of the Declaration of Helsinki. All applicable institutional and governmental regulations concerning the ethical use of human volunteers were followed during this study.

\section{Identification of controls and eligibility}

Contact lens-wearing community controls without microbial keratitis were identified from either contact lens practitioners or recruited from community venues. Three nonmatched controls were identified per case. Controls completed an identical questionnaire that was either selfadministered or completed during a face-to-face interview with interviewers not associated with their eye care.

Eligible contact lens wearers were individuals aged 14-67 years who had worn their lenses in the 4 weeks before the event (cases) or before the survey (controls) for the correction of low refractive errors. Patients with keratoconus, aphakia, or those wearing bandage contact lenses were excluded from this study.

\section{Study definitions}

Study definitions specific to this study are described below. Other definitions are common to previously published studies and have been described elsewhere. ${ }^{13}$

\section{Positive culture}

Corneal specimens were cultured in both aerobic and anaerobic conditions utilising a variety of culture media. A positive bacterial culture was defined as successful isolation of any organism from a specimen in two or more different culture media.

\section{Overnight wear}

Contact lens wearers reporting overnight wear were categorised into one of two groups; either occasional overnight contact lens wearers (reporting overnight wear of less than one night per week) or regular overnight contact lens wearers (one or more nights per week). Participants who did not report any overnight wear were categorised as 'strict daily-wear users'.

\section{Socioeconomic status}

Socioeconomic status (SES) was included as a potential risk factor. The International Standard Classification of Occupations (ISCO-88) was considered as a surrogate measure of SES. Study participants were categorised into higher-skilled (ISCO 1-3) and lower-skilled occupations 
(ISCO 4-9). In participants who were either schooling or conscripted into compulsory national service, their parents' highest rated occupation was used as in lieu of their occupations.

Education levels were also considered a potential surrogate measure for SES. Participants were categorised according to their years of education and highest academic qualifications received. Those indicating uppersecondary or lower as the highest level of education achieved were grouped together, whereas those with diplomas, professional, or tertiary-level qualifications were placed in another category. Participants who did not fall into either category were classified as 'others'.

\section{Hand hygiene}

Hand hygiene habits before handling of contact lenses were examined. Participants were placed in one of three categories: those who washed and dried their hands, only washed their hands without drying, and participants that neither washed nor dried their hands before handling their contact lenses.

\section{Statistical analysis}

Microbial keratitis was the outcome of interest in this study. Potential risk factors identified were tested for statistical significance with univariate analysis using the $\chi^{2}$ test of independence for proportions. Variables with a $P<0.2$ on univariate analysis were further evaluated in a multivariate model. In this model, point estimates of odds ratios were derived utilising binomial logistic regression analysis. These results were subsequently used to determine the degree of association between factors.

Risk factors considered in the construction of the final multivariate model have been listed in Table 1. Variables retained in the multivariate model were factors that either led to an improvement in the $\chi^{2}$ value or identified as confounding factors. Interactions between variables were also examined and included in the model if statistically significant $(P<0.05)$. The strength of association between significant factors is represented by the odds ratios and confidence intervals. All statistical analyses were performed using SPSS software (version 21.0, SPSS Inc., Chicago, IL, USA).

Other statistical tests used in our analysis include the Hosmer-Lemeshow test and variance inflation factor (VIF) analysis. Nagelkerke $R^{2}$ value was also calculated. Furthermore, univariate and combined population attributable risk percentage (PAR\%) were also calculated to evaluate the disease load associated with each risk factor. These permit an estimation of the number of cases that could be eliminated if a particular risk factor was removed.

\section{Results}

A total of 58 cases of microbial keratitis and 152 controls were identified. All participants were included in the final analysis. Demographic data and information on lens types and wear schedule for this cohort of patients have been summarised in Tables 2 and 3.

\section{Univariate analysis}

The racial composition of our study cohort was compared against Singaporean population census data in 2010 from the Singapore Department of Statistics. ${ }^{18}$ Chinese were underrepresented whereas Malays were overrepresented within the cases when compared with both controls and census data. The differences in racial distribution between cases and controls were statistically significant $(P=0.008)$.

Individuals between the ages of 14 and 24 years were also overrepresented among controls. Correspondingly, there appeared to be a greater proportion of 25-44-yearold individuals among cases compared with controls $(P<0.001)$.

In analysing the educational qualifications of our cohort, a larger proportion of cases were found to have a diploma, university, masters or doctorate degree, or other professional qualifications compared with controls $(P=0.100)$.

In terms of lens wear history, the majority of cases had worn contact lenses for $\geq 5$ years compared with controls $(P<0.001)$. In comparison with controls, a significant proportion of cases comprised frequent contact lens users, using their lenses for $>4$ days each week $(P<0.001)$.

A larger percentage of controls wore contact lenses for less than a day each week compared with cases. Cases were more likely to wear lenses overnight for $\geq 2$ nights $(P=0.020)$.

A greater proportion of cases, compared with controls, used planned replacement lenses, defined as weekly, fortnightly, and monthly replacements. Monthly replacement was the most common modality $(P<0.001)$. Correspondingly, there was a smaller proportion of daily disposable lens wearers among cases compared with controls $(P=0.006)$.

Lenses were also categorised and analysed based on their attributes. A larger proportion of cases compared with controls wore silicone hydrogel lenses and cosmetic (lenses to change eye colour) lenses, although this consisted of a small proportion of the total study cohort $(P=0.001)$.

Other significant risk factors identified, when comparing cases with controls, include certain multipurpose solution types $(P<0.001)$, water exposure resulting from activities such as showering while wearing lenses $(P=0.008)$, and poor hand hygiene $(P=0.002)$. 
Table 2 Demographic risk factors

\begin{tabular}{|c|c|c|c|c|}
\hline \multirow[t]{2}{*}{ Risk factors } & \multicolumn{2}{|c|}{$\begin{array}{c}\text { Cases and control } \\
\text { groups }(\%)\end{array}$} & \multirow[t]{2}{*}{$\begin{array}{c}2010 \text { Census Data, } \\
\text { '000 (\%) }\end{array}$} & \multirow[t]{2}{*}{ P-value } \\
\hline & $\begin{array}{c}\text { Microbial keratitis, } \\
\mathrm{n}=58\end{array}$ & $\begin{array}{c}\text { Controls, } \\
\mathrm{n}=152\end{array}$ & & \\
\hline Gender & & & & 0.481 \\
\hline Male & $26(44.8)$ & $60(39.5)$ & $1861.1(49.3)$ & \\
\hline Female & $32(55.2)$ & $92(60.5)$ & $1910.6(50.7)$ & \\
\hline Race & & & & 0.008 \\
\hline Chinese & $37(63.8)$ & $129(84.9)$ & $2794(74.1)$ & \\
\hline Malay & $12(20.7)$ & $13(8.6)$ & $503.9(13.4)$ & \\
\hline Indian & $6(10.3)$ & $5(3.3)$ & $348.1(9.7)$ & \\
\hline Others & $3(5.2)$ & $5(3.3)$ & $125.8(3.3)$ & \\
\hline Age & & & & $<0.001$ \\
\hline $14-24$ & $24(41.4)$ & $101(66.9)$ & $30.9 \%$ & \\
\hline $25-44$ & $33(56.96)$ & $35(23.2)$ & $31.8 \%$ & \\
\hline $45-67$ & $1(1.7)$ & $15(9.9)$ & $37.3 \%$ & \\
\hline ISCO classification & & & & 0.977 \\
\hline ISCO 1-3 & $36(73.5)$ & $98(73.7)$ & & \\
\hline ISCO 4-9 & $13(26.5)$ & $35(26.3)$ & & \\
\hline Education & & & & 0.100 \\
\hline Lower primary to upper secondary & $17(29.3)$ & $33(21.7)$ & & \\
\hline Diplomas, university degrees, and other professional qualifications & $38(65.5)$ & $84(55.3)$ & & \\
\hline Others & $3(5.2)$ & $35(23.0)$ & & \\
\hline
\end{tabular}

Abbreviation: ISCO, International Standard Classification of Occupation.

\section{Microbiology results}

Corneal specimens were obtained for microbiological analysis when possible. Data were available for 46 cases. Of these, missing data were reported for 16 patients and they were excluded from the final analysis. A summary of the microbiology results is shown in Table 4.

Out of the remaining 30 cases, 14 positive corneal culture results $(46.7 \%)$ were identified (Table 4$)$. In all, 12 cultures were positive for Pseudomonas aeruginosa, 1 culture positive for Serratia sp., and 1 culture positive for coagulase-negative Staphylococcus.

\section{Multivariate analysis}

Independent risk factors are shown in Table 5. After adjusting for risk factors identified on univariate analysis, Chinese were found to be at a 7 times lower risk of developing microbial keratitis compared with other races. Those aged between 25 and 44 years were 3 times more likely to develop microbial keratitis compared with younger wearers. Not washing hands before lens handling was associated with a 13-fold increase in risk, whereas occasional overnight wear was found to increase the risk of developing microbial keratitis by fourfold. Patients with microbial keratitis were also more likely to use multipurpose solution A (a sterile, isotonic, buffered solution, preserved with polyhexamethylene biguanide $(0.0001 \%)$, a phosphate buffer, poloxamer 237 , edetate disodium, sodium chloride, potassium chloride, and purified water) when compared with hydrogen peroxide.

The Hosmer-Lemeshow test for logistic regression was performed, demonstrating the goodness of fit of the multivariate model $(P=0.506)$. The Nagelkerke $R^{2}$ value indicated that this multivariate model accounted for $\sim 49.0 \%$ of the variance. VIF analysis was also performed and multicollinearity was not detected, with the VIF not exceeding 3 in any of our analyses.

The univariate $\mathrm{PAR} \%$ for all independent, modifiable risk factors is shown in Table 5. The combined PAR\% for occasional overnight wear, not washing of hands, and use of MPS $\mathrm{A}$ is $81.8 \%$.

\section{Discussion}

This is the first study to measure the risks of all contact lens-related microbial keratitis in Singapore. Microbial keratitis is a disease associated with significant morbidity and identification of modifiable risk factors is important in the ongoing management and prevention of this disease. A cross-sectional survey performed by Lee et al ${ }^{19}$ has suggested that $\sim 9 \%$ of the Singaporean population are contact lens wearers. Extrapolating from 2010 
Table 3 Contact lens-related factors associated with microbial keratitis

\begin{tabular}{|c|c|c|c|}
\hline & \multicolumn{2}{|c|}{ Microbial keratitis and control groups (\%) } & \multirow[t]{2}{*}{ P-value } \\
\hline & Microbial keratitis, $\mathrm{n}=58$ & Controls, $\mathrm{n}=152$ & \\
\hline Duration of lens wear & & & $<0.001$ \\
\hline 1-6 Months & $3(5.2)$ & $21(13.9)$ & \\
\hline 7 Months to 5 years & $18(31.0)$ & $79(52.3)$ & \\
\hline$\geq 6$ Years & $37(63.8)$ & $51(33.8)$ & \\
\hline Wear schedule & & & 0.005 \\
\hline$<1$ Day & $1(1.7)$ & $30(19.9)$ & \\
\hline 1-4 Days & $14(24.1)$ & $41(27.2)$ & \\
\hline$>4$ Days & $43(74.1)$ & $80(53.0)$ & \\
\hline Overnight wear & & & 0.020 \\
\hline Strict DW & $43(74.1)$ & $136(89.5)$ & \\
\hline Occasional ON & $14(24.1)$ & $15(9.9)$ & \\
\hline Regular ON & $1(1.7)$ & $1(0.7)$ & \\
\hline Lens replacement & & & $<0.001$ \\
\hline Daily & $6(10.3)$ & $43(28.3)$ & \\
\hline Weekly, fortnightly, or monthly & $51(87.9)$ & $89(58.6)$ & \\
\hline Yearly & $1(1.7)$ & $20(13.2)$ & \\
\hline \multicolumn{4}{|l|}{ Lens types } \\
\hline Daily disposable soft lenses & $11(20.0)$ & $43(28.3)$ & 0.001 \\
\hline Silicone hydrogel & $14(25.5)$ & $25(16.4)$ & \\
\hline Non-silicone hydrogel & $25(45.5)$ & $64(42.1)$ & \\
\hline Cosmetic & $4(7.3)$ & $0(0)$ & \\
\hline Conventional & $0(0)$ & $16(10.5)$ & \\
\hline Rigid lenses & $1(1.8)$ & $4(2.6)$ & \\
\hline Lens care solutions & & & $<0.001$ \\
\hline Hydrogen peroxide solutions & $1(1.9)$ & $9(8.0)$ & \\
\hline MPS A & $26(50.0)$ & $16(14.3)$ & \\
\hline Other MPS & $25(48.1)$ & $87(77.7)$ & \\
\hline \multicolumn{4}{|l|}{ Water exposure } \\
\hline Showering & & & 0.008 \\
\hline Not showering with lenses & $29(50)$ & $106(70.7)$ & \\
\hline Showering with lenses & $28(48.3)$ & $44(29.3)$ & \\
\hline \multicolumn{4}{|l|}{ Hand hygiene } \\
\hline Washing/drying hands before handling CL & & & 0.002 \\
\hline Wash and dry & $23(39.7)$ & $94(61.8)$ & \\
\hline Wash only & $28(48.3)$ & $54(35.5)$ & \\
\hline Neither wash nor dry & 7 (12.1) & $4(2.6)$ & \\
\hline Hygiene score & & & 0.268 \\
\hline Poor & $13(24.1)$ & $17(14.9)$ & \\
\hline Moderate & $16(29.6)$ & $30(26.3)$ & \\
\hline Good & $7(13.0)$ & $12(10.5)$ & \\
\hline Excellent & $18(33.3)$ & $55(48.2)$ & \\
\hline
\end{tabular}

Abbreviations: DW, daily wear; MPS, multipurpose solution; ON, overnight.

population census data, ${ }^{18}$ this translates to $\sim 456903$ contact lens wearers during the study period and this would result in $\sim 200$ wearers developing microbial keratitis each year in Singapore. ${ }^{6}$ Given access to primary eye care in Singapore, these acute cases would be managed through tertiary care centres and this approach is likely to capture a representative sample of cases.

Given the unique sociocultural, environmental, and climate conditions, along with differences in contact lens prescribing practice and eye care availability, it is 
conceivable that risk factors of contact lens wearers in Singapore may differ from those previously reported.

Age and race were important sociodemographic factors. Chinese were found to be at a lower risk of developing microbial keratitis compared with other races. This is consistent with findings in a Singaporean fungal keratitis case-control study that found that Chinese wearers were at a lower risk of developing Fusarium keratitis. ${ }^{20}$ A multicentre cross-sectional survey of contact lens complications in Singapore found that $77 \%$ of patients who presented with complications were Chinese. ${ }^{21}$ However, no disease-free lens wearing controls were included in the published study.

Table 4 Positive corneal cultures

\begin{tabular}{lc}
\hline Microbial organism cultured & $\begin{array}{c}\text { Number of positive } \\
\text { cultures (\%) }\end{array}$ \\
\hline Pseudomonas aeruginosa & $12(85.7)$ \\
Serratia sp. & $1(7.1)$ \\
Coagulase-negative Staphylococcus & $1(7.1)$ \\
Total & 14 \\
\hline
\end{tabular}

The differences in risk profile among racial subgroups may be attributed to disparities in health-related quality of life. Clinically significant differences have been demonstrated among various racial groups in Singapore, ${ }^{22}$ where Chinese scored highest in five out of eight scales of the Short Form Health Survey (SF-36) compared with their Malay and Indian counterparts. This instrument is a validated, self-administered questionnaire that measured health-related quality of life across various domains, with higher scores indicative of better-perceived health.

Although socioeconomic class was not identified as an independent risk factor, race may be considered a surrogate measure, as the most recent population census demonstrated significant differences in average monthly household incomes between different racial groups. ${ }^{23}$ Both Chinese and Indian households demonstrated similar earning power that was higher than the average monthly Malay household income. This is consistent with study findings reporting an association between low socioeconomic class and increased risk of keratitis. ${ }^{9}$ However, findings in other studies shown higher socioeconomic class to be linked to an increased risk of

Table 5 Independent risk factors for microbial keratitis

\begin{tabular}{|c|c|c|c|c|}
\hline Risk factor & Odds ratio $(O R)$ & $95 \% \mathrm{CI}$ & P-value & $P A R \%$ \\
\hline \multicolumn{5}{|l|}{ Race } \\
\hline Chinese & 1.00 (Referent) & & & \\
\hline Other races & 7.02 & $2.32-21.29$ & 0.001 & $83.6 \%$ \\
\hline \multicolumn{5}{|l|}{ Age (years) } \\
\hline $14-24$ & 1.00 (Referent) & & & \\
\hline $25-44$ & 3.2 & $1.07-9.57$ & 0.037 & $33.8 \%$ \\
\hline $45-67$ & 0.25 & $0.02-2.73$ & $0.255^{\mathrm{a}}$ & \\
\hline \multicolumn{5}{|l|}{ Duration } \\
\hline 1-6 Months & 1.00 (Referent) & & & \\
\hline 7 Months to 5 years & 0.43 & $0.08-2.29$ & $0.320^{\mathrm{a}}$ & NA \\
\hline$\geq 6$ Years & 1.7 & $0.31-9.52$ & $0.544^{\mathrm{a}}$ & \\
\hline \multicolumn{5}{|l|}{ Overnight wear } \\
\hline Strict daily wear & 1.00 (Referent) & & & \\
\hline Occasional overnight wear & 4.26 & $1.18-15.37$ & 0.027 & $24.3 \%$ \\
\hline Overnight wear & 1.89 & $0.09-40.80$ & $0.684^{\mathrm{a}}$ & \\
\hline \multicolumn{5}{|l|}{ Washing and drying of hands } \\
\hline Wash and dry & 1.00 (Referent) & & & \\
\hline Wash only & 1.92 & $0.70-5.28$ & $0.207^{\mathrm{a}}$ & \\
\hline Not washing & 12.77 & $1.93-84.78$ & 0.008 & $23.7 \%$ \\
\hline \multicolumn{5}{|l|}{ Multipurpose solutions } \\
\hline Hydrogen peroxide solutions & 1.00 (Referent) & & & \\
\hline MPS A & 16.2 & $1.52-174.03$ & 0.021 & $68.5 \%$ \\
\hline Other MPS & 2.3 & $0.23-22.02$ & $0.482^{\mathrm{a}}$ & \\
\hline
\end{tabular}

Abbreviations: CI, confidence interval; MPS, multipurpose solution.

Individual univariate population attributable risk percentages (PAR\%) may add up to $>100 \%$ because exposures are not mutually exclusive. ${ }^{\text {a }}$ Not significantly different. 
microbial keratitis. ${ }^{6,8,16}$ Further research is required to establish the association between race, SES, and microbial keratitis and any potential confounders.

Those aged between 25 and 44 years had an increased risk of developing microbial keratitis compared with younger wearers aged between 14 and 24 years. Previous studies have described a higher risk in younger age groups, 3,13 and this finding is unexpected.

Certain contact lens wear practices have also been associated with an increased risk of developing contact lens-related microbial keratitis. Occasional overnight wear was independently associated with an increased risk of developing contact lens-related microbial keratitis. This was consistent with previous studies of contact lensrelated microbial keratitis. ${ }^{6,13}$ However, regular overnight lens wear was not identified as a significant risk factor in our multivariate model, likely reflecting the limited penetrance of extended wear in Singapore. This finding was consistent with 2012 fitting data from Singapore demonstrating limited uptake of extended wear soft contact lenses (personal communication, Morgan and Woods, 2012).

Not washing of hands before handling contact lenses was identified as a risk factor on multivariate analysis, accounting for $24 \%$ of the disease load. Poor hand hygiene has been consistently previously reported as a risk factor. ${ }^{6,13,16}$ Poor hand hygiene may result in the introduction of possible pathogens during lens insertion and lens manipulation during wear and microbial contamination of storage cases. ${ }^{24}$ This is an important compliance message both for the initial dispensing of contact lenses and during aftercare visits.

The use of MPS A was associated with an increased risk of developing contact lens-related microbial keratitis, accounting for $69 \%$ of the disease load. This represents a very high risk to contact lens users and was independent of other hygiene and compliance factors. Similar findings have been described in a separate study examining risk factors in an Australian population with moderate and severe contact lens-related microbial keratitis in daily wear lens users. ${ }^{16}$ Previously published studies have also reported associations between certain multipurpose solution types and Fusarium and Acanthamoeba outbreaks of contact lens-related microbial keratitis. ${ }^{25-27}$

Certain polymeric biguanide-based care solutions have been demonstrated to be less effective against bacterial biofilms in comparison with hydrogen peroxide or polyquaternium-based solutions. ${ }^{28}$ In addition, reduction in microbial efficacy of this product has been reported when used for the storage of contact lenses in lens cases over a prolonged period of time. ${ }^{29}$ The increased risk identified with the use of MPS A in both clinical and laboratory studies may suggest failure to achieve a suitable level of disinfection efficacy particularly in the hands of the wearer.

Other compliance factors, such as case hygiene practice and disinfection frequency, were not identified as significant factors in this study, and this was unexpected given the strong association with microbial keratitis in daily wear use.

Microbiological findings in this study are similar to those reported by Dart et $a{ }^{13}$ and are broadly consistent with existing literature reports of $P$. aeruginosa as the most prevalent organism isolated in $50-60 \%$ of culture-proven contact lens-related disease. ${ }^{16,30-33}$ Environmental organisms were recovered in all but one of the culturepositive corneal samples and, although these numbers are low, this finding would be consistent with higher recovery of environmental causative organisms in tropical climate zones. ${ }^{15} \mathrm{P}$. aeruginosa is a common environmental pathogen that is able to survive in contact lens storage cases. ${ }^{34,35}$ It demonstrates a greater ability to adhere to contact lenses compared with other bacteria, ${ }^{36}$ and is associated with both a more severe disease spectrum and poorer visual prognosis. ${ }^{14}$ Our study findings therefore highlight the importance of prompt identification and management of microbial keratitis to limit disease severity and optimise patient outcomes.

Limitations to this study have been recognised. The multivariate model accounts for approximately half of the variance in the data. This suggests that other healthrelated or behavioural risk factors not surveyed in this study are likely to be important.

In addition, the selection of controls to minimise selection bias in case-control studies is challenging. Potential sampling biases from techniques employed in recruiting controls may have been encountered in this study. Differences between the composition of our control group and 2010 census data were also identified. Chinese were overrepresented, whereas Malays and Indians were underrepresented in our control population. However, there is limited information on the contact lens-related population characteristics in Singapore. Furthermore, the control group in this study is consistent with the typical profile of contact lens wearers elsewhere. ${ }^{37}$

In conclusion, our findings have implications for the prevention and management of microbial keratitis in Singapore. Contact lens-related microbial keratitis was associated with race, wearer age, occasional overnight wear, poor hand hygiene, and lens care solution type. The high PAR\% for infection associated with a particular solution product available in Singapore is of significant concern. Modifiable risk factors may be amenable to education of contact lens wearers by eye care providers. Previous studies have indicated more severe disease because of environmental organisms occurring more commonly in frequent replacement daily wear users 
compared with daily disposable users. Given the high rate of environmental organisms recovered, daily disposable contact lenses may be a safer alternative to reduce morbidity. 6,38

\section{Summary}

What was known before

- Independent risk factors for contact lens-related microbial keratitis, including poor hand hygiene, occasional overnight wear, and type of lens care solution, have been identified in previous studies.

What this study adds

- This is the first study to measure risks of contact lensrelated microbial keratitis in Singapore. Differences in risk profiles between ethnicities were identified in our multivariate model.

\section{Conflict of interest}

The authors declare no conflict of interest.

\section{Acknowledgements}

We thank Thomas Naduvilath for statistical advice. This study was supported by an unrestricted grant from Alcon. This study was presented at ARVO 2013 in Seattle, USA.

\section{References}

1 Stapleton F, Keay L, Jalbert I, Cole N. The epidemiology of contact lens related infiltrates. Optom Vis Sci 2007; 84(4): 257-272.

2 Poggio EC, Glynn RJ, Schein OD, Seddon JM, Shannon MJ, Scardino VA et al. The incidence of ulcerative keratitis among users of daily-wear and extended-wear soft contact lenses. New Engl J Med 1989; 321(12): 779-783.

3 Cheng KH, Leung SL, Hoekman HW, Beekhuis WH, Mulder PG, Geerards AJ et al. Incidence of contact-lensassociated microbial keratitis and its related morbidity. Lancet 1999; 354(9174): 181-185.

4 Lam DS, Houang E, Fan DS, Lyon D, Seal D, Wong E. Incidence and risk factors for microbial keratitis in Hong Kong: comparison with Europe and North America. Eye 2002; 16(5): 608-618.

5 Morgan PB, Efron N, Hill EA, Raynor MK, Whiting MA, Tullo AB. Incidence of keratitis of varying severity among contact lens wearers. Br J Ophthalmol 2005; 89(4): 430-436.

6 Stapleton F, Keay L, Edwards K, Naduvilath T, Dart JK, Brian $\mathrm{G}$ et al. The incidence of contact lens-related microbial keratitis in Australia. Ophthalmology 2008; 115(10): 1655-1662.

7 Dart JK, Stapleton F, Minassian D. Contact lenses and other risk factors in microbial keratitis. Lancet 1991; 338(8768): 650-653.

8 Stapleton F, Dart JK, Minassian D. Risk factors with contact lens related suppurative keratitis. CLAO J 1993; 19(4): 204-210.
9 Schein OD, Glynn RJ, Poggio EC, Seddon JM, Kenyon KR. The relative risk of ulcerative keratitis among users of daily-wear and extended-wear soft contact lenses. A casecontrol study. Microbial Keratitis Study Group. New Engl J Med 1989; 321(12): 773-778.

10 Radford CF, Minassian DC, Dart JK. Disposable contact lens use as a risk factor for microbial keratitis. $\mathrm{Br} J$ Ophthalmol 1998; 82(11): 1272-1275.

11 Morgan PB, Efron N, Brennan NA, Hill EA, Raynor MK, Tullo AB. Risk factors for the development of corneal infiltrative events associated with contact lens wear. Invest Ophthalmol Vis Sci 2005; 46(9): 3136-3143.

12 Keay L, Edwards K, Stapleton F. Signs, symptoms, and comorbidities in contact lens-related microbial keratitis. Optom Vis Sci 2009; 86(7): 803-809.

13 Dart JK, Radford CF, Minassian D, Verma S, Stapleton F. Risk factors for microbial keratitis with contemporary contact lenses: a case-control study. Ophthalmology 2008; 115 (10): 1647-1654.

14 Keay L, Edwards K, Naduvilath T, Forde K, Stapleton F. Factors affecting the morbidity of contact lens-related microbial keratitis: a population study. Invest Ophthalmol Vis Sci 2006; 47(10): 4302-4308.

15 Stapleton F, Keay LJ, Sanfilippo PG, Katiyar S, Edwards KP, Naduvilath T. Relationship between climate, disease severity, and causative organism for contact lens-associated microbial keratitis in Australia. Am J Ophthalmol 2007; 144(5): 690-698.

16 Stapleton F, Edwards K, Keay L, Naduvilath T, Dart JK, Brian $\mathrm{G}$ et al. Risk factors for moderate and severe microbial keratitis in daily wear contact lens users. Ophthalmology 2012; 119(8): 1516-1521.

17 Radford CF, Minassian D, Dart JK, Stapleton F, Verma S. Risk factors for nonulcerative contact lens complications in an ophthalmic accident and emergency department: a case-control study. Ophthalmology 2009; 116(3): 385-392.

18 Kim WW. Census of Population 2010: Advance Census Release. Department of Statistics, Ministry of Trade and Industry: Singapore, 2011.

19 Lee YC, Lim CW, Saw SM, Koh D. The prevalence and pattern of contact lens use in a Singapore community. CLAO J 2000; 26(1): 21-25.

20 Saw SM, Ooi PL, Tan DT, Khor WB, Fong CW, Lim J et al. Risk factors for contact lens-related fusarium keratitis: a case-control study in Singapore. Arch Ophthalmol 2007; 125(5): 611-617.

21 Teo L, Lim L, Tan DTH, Chan T-K, Jap A, Ming LH. A survey of contact lens complications in Singapore. Eye Contact Lens 2011; 37(1): 16-19.

22 Thumboo J, Fong KY, Machin D, Chan SP, Soh CH, Leong $\mathrm{KH}$ et al. Quality of life in an urban Asian population: the impact of ethnicity and socio-economic status. Soc Sci Med 2003; 56(8): 1761-1772.

23 Kim WWCensus of Population 2010 Statistical Release 2: Households and HousingDepartment of Statistics, Ministry of Trade and Industry, Republic of Singapore: Singapore, 2011.

24 McMonnies CW. Hand hygiene prior to contact lens handling is problematical. Cont Lens Anterior Eye 2012; 35(2): 65-70.

25 Joslin CE, Tu EY, Shoff ME, Booton GC, Fuerst PA, McMahon TT et al. The association of contact lens solution use and Acanthamoeba keratitis. Am J Ophthalmol 2007; 144(2): 169-180. 
26 Chang DC, Grant GB, O’Donnell K, Wannemuehler KA, Noble-Wang J, Rao CY et al. Multistate outbreak of Fusarium keratitis associated with use of a contact lens solution. JAMA 2006; 296(8): 953-963.

27 Khor WB, Aung T, Saw SM, Wong TY, Tambyah PA, Tan AL et al. An outbreak of Fusarium keratitis associated with contact lens wear in Singapore. JAMA 2006; 295(24): 2867-2873.

28 Szczotka-Flynn LB, Imamura Y, Chandra J, Yu C, Mukherjee PK, Pearlman E et al. Increased resistance of contact lens-related bacterial biofilms to antimicrobial activity of soft contact lens care solutions. Cornea 2009; 28(8): 918-926.

29 Dannelly HK, Waworuntu RV. Effectiveness of contact lens disinfectants after lens storage. Eye Contact Lens 2004; 30(3): 163-165.

30 Bharathi MJ, Ramakrishnan R, Shivakumar C, Meenakshi R, Lionalraj D. Etiology and antibacterial susceptibility pattern of community-acquired bacterial ocular infections in a tertiary eye care hospital in south India. Indian J Ophthalmol 2010; 58(6): 497-507.

31 Orlans H, Hornby S, Bowler I. In vitro antibiotic susceptibility patterns of bacterial keratitis isolates in Oxford, UK: a 10-year review. Eye 2011; 25(4): 489-493.

32 van der Meulen IJ, van Rooij J, Nieuwendaal CP, Van Cleijnenbreugel H, Geerards AJ, Remeijer L. Age-related risk factors, culture outcomes, and prognosis in patients admitted with infectious keratitis to two Dutch tertiary referral centers. Cornea 2008; 27(5): 539-544.

33 Shalchi Z, Gurbaxani A, Baker M, Nash J. Antibiotic resistance in microbial keratitis: ten-year experience of corneal scrapes in the United Kingdom. Ophthalmology 2011; 118(11): 2161-2165.

34 Gray TB, Cursons R, Sherwan JF, Rose PR. Acanthamoeba, bacterial, and fungal contamination of contact lens storage cases. Br J Ophthalmol 1995; 79(6): 601-605.

35 Willcox MD, Carnt N, Diec J, Naduvilath T, Evans V, Stapleton $\mathrm{F}$ et al. Contact lens case contamination during daily wear of silicone hydrogels. Optom Vis Sci 2010; 87(7): 456-464.

36 Vijay AK, Zhu H, Ozkan J, Wu D, Masoudi S, Bandara R et al. Bacterial adhesion to unworn and worn silicone hydrogel lenses. Optom Vis Sci 2012; 89(8): 1095-1106.

37 Morgan PB, Efron N, Helland M, Itoi M, Jones D, Nichols JJ et al. Demographics of international contact lens prescribing. Cont Lens Anterior Eye 2010; 33(1): 27-29.

38 Stapleton F, Naduvilath T, Keay LJ, Radford CF, Dart JK, Edwards $\mathrm{K}$ et al. Risk factors for microbial keratitis in daily disposable contact lens wear. Invest Ophthalmol Vis Sci 2010; 51(e-abstract 1305). 